\title{
DEVELOPMENT AND APPLICATIONS OF A STAGE STACKING PROCEDURE
}

\author{
Sameer Kulkarni \\ NASA Glenn Research Center \\ Cleveland, Ohio, USA
}

\author{
Mark L. Celestina \\ NASA Glenn Research Center \\ Cleveland, Ohio, USA
}

\author{
John J. Adamczyk \\ NASA Glenn Research Center, DRA \\ Cleveland, Ohio, USA
}

\begin{abstract}
The preliminary design of multistage axial compressors in gas turbine engines is typically accomplished with mean-line methods. These methods, which rely on empirical correlations, estimate compressor performance well near the design point, but may become less reliable off-design. For land-based applications of gas turbine engines, off-design performance estimates are becoming increasingly important, as turbine plant operators desire peaking or load-following capabilities and hot-day operability. The current work develops a onedimensional stage stacking procedure. This includes a newlydefined blockage term, which is used to estimate the off-design performance and operability range of a 13-stage axial compressor. The new blockage term is defined to give mathematical closure on static pressure, and values of blockage are shown to collapse to a curve as functions of stage inlet flow coefficient and corrected speed. Utility of the stage stacking procedure is demonstrated by estimation of the minimum corrected speed which allows stable operation of the compressor. Further utility of the stage stacking procedure is demonstrated with a bleed sensitivity study, which estimates a bleed schedule to expand the compressor's operating range.
\end{abstract}

\section{NOMENCLATURE} Roman Symbols

$A_{a n} \quad$ Geometrical annulus area, $\mathrm{m}^{2}$

$A_{e f} \quad$ Effective flow area, $\mathrm{m}^{2}$

$c_{p} \quad$ Specific heat at constant pressure, $\mathrm{J} /(\mathrm{kg} \mathrm{K})$

$k_{b} \quad$ Effective blockage factor

M Mach number

in $\quad$ Mass flow rate, $\mathrm{kg} / \mathrm{s}$

$N_{s} \quad$ Shaft rotational speed
$N_{c} \quad$ Corrected rotational speed

$P \quad$ Total (stagnation) pressure, $\mathrm{Pa}$

$P_{s l} \quad$ NASA standard sea-level pressure $=101,315 \mathrm{~Pa}$

$p_{s} \quad$ Static pressure, $\mathrm{Pa}$

$R \quad$ Specific gas constant $=287.04 \mathrm{~J} /(\mathrm{kg} \mathrm{K})$

$r_{m} \quad$ Midspan radius, $\mathrm{m}$

$T \quad$ Total (stagnation) temperature, $\mathrm{K}$

$T_{s l} \quad$ NASA standard sea-level temperature $=288.17 \mathrm{~K}$

$U_{m} \quad$ Midspan rotor speed, $\mathrm{m} / \mathrm{s}$

$V_{z} \quad$ Axial flow velocity, $\mathrm{m} / \mathrm{s}$

\section{Greek Symbols}

a Absolute flow angle, deg

$\gamma \quad$ Ratio of specific heats

$\eta \quad$ Adiabatic efficiency

$\varphi \quad$ Flow coefficient

$\psi \quad$ Ideal work coefficient

(1) Shaft angular frequency, $\mathrm{rad} / \mathrm{s}$

\section{Subscripts}

$1 \quad$ Stage inlet station

$2 \quad$ Stage exit station

\section{INTRODUCTION}

Compressors in modern industrial gas turbine engines used for power generation are designed to operate continuously at a physical shaft speed corresponding to the utility frequency of either $50 \mathrm{~Hz}$ or $60 \mathrm{~Hz}$. Industrial engines generally operate continuously for months while generating a fixed power output, and only require inspection and maintenance every few years. Typical maintenance intervals for these "baseload" engines are on the order of 25,000 hours or 800 starts [1]. Due to the

This material is declared a work of the U.S. Government and is not subject to copyright protection in the United States. Approved for public release; distribution is unlimited. 
operation of these engines at a fixed shaft speed, stability issues arise. The engine power output requirement and shaft speed are fixed, whereas the compressor inlet temperature varies according to the local climate, causing a change in the corrected speed defined as

$$
N_{c}=\frac{N_{s}}{\sqrt{T / T_{s l}}}
$$

Here $N_{s}$ is the physical shaft speed, $T$ is the ambient, or compressor inlet total temperature, and $T_{s l}$ is the NASA standard sea-level temperature of $288.17 \mathrm{~K}$. It has been reported that industrial gas turbine engine power output can drop by as much as $0.9 \%$ for every $1 \mathrm{~K}$ rise in ambient temperature [2]. Care must be taken during the preliminary design of these compressors to ensure stable operation across the expected range of ambient temperatures.

Besides the design requirements imposed by hot day operation, additional requirements are emerging due to the expected increase of renewable energy sources in the coming years [1]. Wind turbines and solar cells provide a varying amount of power dictated by local weather conditions. During cloudy days or days with low wind speed, gas turbine engines may be used to supplement power, especially during the hours when consumption demand, and thus the price of electricity, is high. This large demand generally exceeds the capacity that can be met by baseload engines, wind turbines, and solar cells. Future gas turbine engines must perform under a cyclic service duty to achieve this "peaking" capability. The future engine must be able to provide a range of power output at fixed shaft speed (as opposed to a fixed power output at fixed shaft speed) so that the engine output can follow demand throughout the day. This effectively requires the engine to be designed for multiple points of operation and presents an engineering challenge in the aerodynamic design of the compressor.

In a preliminary aerodynamic design system, empirically based methods, referred to as mean-line or throughflow models, are used by engine manufacturers to establish an initial geometry for a new compressor [3-5]. These techniques make use of the breadth of experience and knowledge accrued from past compressor designs in the form of empirical or semiempirical correlations to estimate parameters of the flow, such as blockage, loss, and deviation. These parameters have a large impact upon the performance and operability of the compressor. These mean-line or throughflow models can estimate the performance of a new compressor design quite well near its design point. However, these methods can become less reliable off-design, or for new designs which differ substantially from previous ones. Off-design performance estimates are becoming more important, as hot-day operation and load-following or peaking capabilities become increasingly desirable for turbine plant operators. This poses a challenge during a preliminary design when critical performance estimates far from the design point are required. Thus, there exists a need for a methodology to generate credible estimates of off-design performance and operability of these compressors during preliminary design.

With the increasing power and availability of computer resources, engine manufacturers can now use three-dimensional computational fluid dynamics (CFD) solvers to examine offdesign operation of a compressor. These solvers rely less on empirical correlations than most mean-line and throughflow methods. A properly validated and calibrated CFD solver can thus provide insight into off-design compressor performance.

The objective of this work is to develop a methodology which leads to credible estimates of aero-operability and aeroperformance of axial multistage compressors at design and at off-design operating points for use in the preliminary design of a compressor whose design parameters are near that of an existing configuration. This objective is accomplished by developing a one-dimensional stage stacking procedure and using it to estimate design and off-design performance and operability limits of a 13-stage axial flow compressor. This compressor is part of an industrial gas turbine engine used for power generation. The stage stacking procedure utilizes stage performance curves which are constructed using flow information extracted from a database of CFD simulations of the compressor. This database is generated using the APNASA CFD code for multistage turbomachinery.

\section{DETAILS OF THE COMPRESSOR AND CFD MODEL}

The focus of the current work is a 13-stage axial flow compressor which is part of an industrial gas turbine engine. The design shaft speed for the compressor is 3600 RPM, corresponding to a $60 \mathrm{~Hz}$ utility frequency. The compressor consists of an inlet guide vane (IGV), 13 rotor-stator stages, and an outlet guide vane $(\mathrm{OGV})$. All 28 blade rows are modeled in the current work. The IGV as well as stators 1, 2, and 3 (referred to as variable guide vanes, or VGVs) have variable stagger angles. The remaining stators and OGV are cantilevered.

A computational grid modeling the compressor was created using the mesh generator detailed by Mulac [6]. This generator produces an H-mesh for each blade row, with common axial and radial coordinates shared between the meshes. The meshes contain 51 nodes in the tangential direction (from the suction side of one blade to the pressure side of the next blade) and 51 nodes in the radial direction (from hub to casing). Each blade is modeled with 61 axial nodes along the chord, from leading edge to trailing edge. The number of axial nodes from the inlet plane to the exit plane of the grid totals 3067 nodes. A meridional view of the compressor flow path is shown in Figure 1.

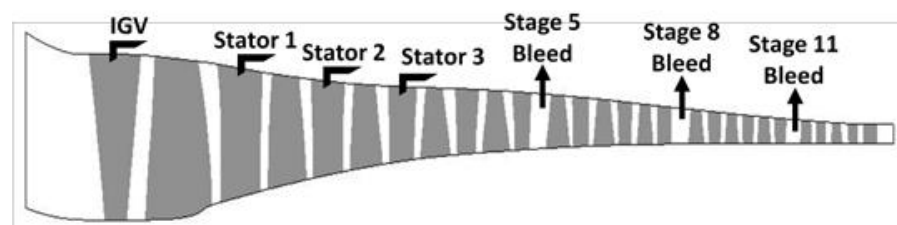

FIGURE 1: MERIDIONAL VIEW OF THE COMPRESSOR

2

This material is declared a work of the U.S. Government and is not subject to copyright protection in the United States. Approved for public release; distribution is unlimited. 
The three VGVs were set to nominal settings, and the IGV was closed $2^{\circ}$ from nominal in order to match the flow rate obtained from previous experimental tests of this compressor. These setting angles were fixed irrespective of simulated operating conditions. Stator platform leakages associated with the IGV and VGVs are not modeled in this work. This is because leakage estimates for this machine were extremely small compared to what is typical in compressors for aircraft engines. There are three casing bleeds in the compressor, located downstream of stators 5,8 , and 11. The removal of mass flow due to these bleeds is accounted for in the CFD code by using an aspiration model. Bleed flow was removed along an axisymmetric ring at the casing, downstream of the relevant stators. At design speed, the bleed flow rates are set to their nominal values. For part-speed operation, bleed rates are scaled by percent corrected speed. The physical geometry of the casing bleeds are not modeled in this work.

CFD simulations of the compressor were generated using the APNASA turbomachinery code. The APNASA code, developed by Adamczyk, et al. [7], solves a set of equations known as the Average-Passage model, derived by Adamczyk [8], based on a sequence of mathematical averaging of the Navier-Stokes equations, continuity equation, energy equation, and the equation of state. The code employs a standard k- $\varepsilon$ turbulence model to close on the Reynolds stress term. Details of the code algorithm and governing equations can be found in [7-10].

An inlet boundary condition was prescribed at the first axial plane of the computational grid, at a location upstream of the IGV leading edge. This inlet boundary condition was specified as a radial profile of total pressure, total temperature, tangential flow angle, and radial flow angle. The inlet profile was obtained from an existing throughflow calculation for this machine, and was fixed irrespective of operating conditions. An exit boundary condition was prescribed at the last axial plane of the computational grid. This exit boundary condition was a static pressure specified at the hub using a simple radial equilibrium model to establish the spanwise flow distribution [7]. The simulation was throttled along a speed line by varying this exit hub static pressure.

The corrected speed was varied by specifying the physical shaft speed, as opposed to changing the inlet temperature. This was done to emulate a series of surge tests that had previously been performed for this compressor. In these experiments, the IGV and VGV settings were fixed and mechanical shaft speed was reduced until the occurrence of a surge event. The reduction in speed was done at a rate such that clearances were minimally affected. It should be noted that this operation differs from routine operation of the engine, in which corrected speed varies according to changes in ambient temperature conditions while the physical shaft speed of the compressor is fixed according to the utility frequency of $60 \mathrm{~Hz}$. The simulation results were compared to a limited set of stator leading edge total pressure and total temperature measurements, and agreement was judged to be satisfactory for the purposes of this work.

\section{STAGE STACKING PROCEDURE}

A one-dimensional procedure for estimating overall compressor performance, known as stage stacking, was described by Robbins and Dugan [11]. This method is frequently used in the preliminary design of axial compressors. The stage stacking procedure requires performance curves for every stage to be available. The performance curves consist of an ideal work coefficient and an adiabatic efficiency which are plotted as a function of stage inlet flow coefficient. The current work expands upon the procedure described by Robbins and Dugan [11] by defining and implementing a blockage term into the stage stacking procedure. If modifying an existing design, the performance curves may be generated from experimental tests. In the current work, the stage performance curves are generated using flow information extracted from CFD simulations, as will be detailed later.

\section{Stage Performance Parameters}

The stage ideal work coefficient $\psi$ is defined as the ideal total enthalpy rise across the stage, non-dimensionalized by midspan wheel speed squared, as in

$$
\psi=\frac{c_{p} T_{s l}\left[\left(\frac{P_{2}}{P_{1}}\right)^{\frac{\gamma_{a v g}-1}{\gamma_{\text {avg }}}}-1\right]}{\left(\frac{U_{m, 1}}{\sqrt{\frac{T_{1}}{T_{s l}}}}\right)^{2}}
$$

In the current work, total temperature $T_{l}$ and total pressures $P_{1}$ and $P_{2}$ are mass averaged values obtained from a CFD derived database. The subscripts 1 and 2 indicate the stage inlet and exit stations, respectively. The NASA standard sea-level total pressure and total temperature, $P_{s l}$ and $T_{s l}$, are $101,315 \mathrm{~Pa}$ and $288.17 \mathrm{~K}$, respectively. The ratio of specific heats used here, $\gamma_{\text {avg }}$, is the arithmetic average of the ratios of specific heat at the stage inlet and stage exit stations, $\gamma_{1}$ and $\gamma_{2}$, respectively. The specific heat at constant pressure $c_{p}$ is given by

$$
c_{p}=R \frac{\gamma_{a v g}}{\gamma_{a v g}-1}
$$

Here, $R$ is the specific gas constant for air, $287.04 \mathrm{~J} /(\mathrm{kg} \mathrm{K})$. The midspan rotor speed at the stage inlet station $U_{m, 1}$ is given by

$$
U_{m, 1}=\omega r_{m, 1}
$$

Here, $\omega$ is the angular frequency of the rotating shaft with units of $\mathrm{rad} / \mathrm{s}$ and $r_{m, 1}$ is the midspan radius at the stage inlet station. Stage adiabatic efficiency $\eta$ is defined as the ratio of the ideal total enthalpy rise to the actual total enthalpy rise, as given by

This material is declared a work of the U.S. Government and is not subject to copyright protection in the United States. Approved for public release; distribution is unlimited. 


$$
\eta=\frac{\left(\frac{P_{2}}{P_{1}}\right)^{\frac{\gamma_{a v g}-1}{\gamma_{a v g}}}-1}{\frac{T_{2}}{T_{1}}-1}
$$

The stage inlet flow coefficient $\varphi_{l}$ is defined as the ratio of corrected axial velocity to corrected midspan rotor speed, as given by

$$
\varphi_{1}=\frac{V_{z, 1} / \sqrt{\frac{T_{1}}{T_{s l}}}}{U_{m, 1} / \sqrt{\frac{T_{1}}{T_{s l}}}}
$$

The corrected axial velocity in Eq. (6) is solved from the following equation from [11]:

$$
\begin{aligned}
& \frac{\dot{m} \sqrt{\frac{T_{1}}{T_{s l}}}}{A_{e f, 1}\left(\frac{P_{1}}{P_{s l}}\right)} \\
& =\frac{V_{z, 1}}{\sqrt{\frac{T_{1}}{T_{s l}}}}\left[1-\left(\frac{V_{z, 1}}{\sqrt{\frac{T_{1}}{T_{s l}}}}\right)^{2} \frac{1}{2 c_{p} T_{s l} \cos ^{2} \alpha_{1}}\right]^{\frac{1}{\gamma_{1}-1}} \frac{P_{s l}}{R T_{s l}}
\end{aligned}
$$

The design value of stage inlet midspan absolute flow angle $\alpha_{1}$ is used here, and $\dot{m}$ is the mass flow rate at the stage inlet station. The value of stage inlet mass flow rate is equal to the compressor inlet mass flow rate, less any applicable flow removal due to bleeds. The current work expands upon the method described in [11] regarding the area term $A_{e f, 1}$ on the left-hand-side of Eq. (7). This term is generally taken to be the geometric annulus area, but more realistic values of corrected axial velocity are achieved if one accounts for flow blockage [11]. In the current work, the term $A_{e f}$ is taken as an effective flow area, defined such that it provides mathematical closure on static pressure $p_{s, l}$ as given by the equations

$$
\begin{aligned}
& \frac{\dot{m} \sqrt{\frac{T_{1}}{T_{s l}}}}{A_{e f, 1}\left(\frac{P_{1}}{P_{S l}}\right)} \\
& =\frac{P_{s l}}{\sqrt{T_{s l}}} \sqrt{\frac{\gamma_{1}}{R}} M_{1} \cos \alpha_{1}\left(1+\frac{\gamma_{1}-1}{2} M_{1}{ }^{2}\right)^{-\left(\frac{\gamma_{1}+1}{2 \gamma_{1}-2}\right)} \\
& \quad \frac{p_{s, 1}}{P_{1}}=\left(1+\frac{\gamma_{1}-1}{2} M_{1}{ }^{2}\right)^{-\left(\frac{\gamma_{1}}{\gamma_{1}-1}\right)}
\end{aligned}
$$

Combining the above equations and solving for $A_{e f, 1}$ gives

$$
=\frac{A_{e f, 1}}{\frac{\dot{m} \sqrt{\frac{T_{1}}{T_{s l}}} /\left(\frac{P_{1}}{P_{S l}}\right) \sqrt{\frac{R}{\gamma_{1}}}}{\sqrt{T_{s l}}\left(\frac{p_{s, 1}}{P_{1}}\right)^{\frac{\gamma_{1}+1}{2 \gamma_{1}}} \cos \alpha_{1} \sqrt{\left\{\left(\frac{p_{S, 1}}{P_{1}}\right)^{-\left(\frac{\gamma_{1}-1}{\gamma_{1}}\right)}-1\right\} \frac{2}{\gamma_{1}-1}}}}
$$

The static pressure term is taken as the area averaged value, extracted from CFD simulations of the compressor, but may alternatively be supplied by experimental tests or by correlations. Using this effective area term, an effective blockage term $k_{b}$ is defined as

$$
k_{b}=1-\frac{A_{e f}}{A_{a n}}
$$

Here $A_{a n}$ is the geometric annulus area. Thus, the following information must be known to estimate compressor performance using the stage stacking procedure developed in the current work:

1. Fully populated performance curves $\left(\psi, \eta, k_{b}\right.$ vs. $\left.\varphi_{1}\right)$ for each stage at the corrected speeds of interest.

2. Geometrical annulus area at each stage inlet and exit station $\left(A_{a n, 1}\right.$ and $\left.A_{a n, 2}\right)$.

3. Midspan radius at each stage inlet and exit station $\left(r_{m, l}\right.$ and $\left.r_{m, 2}\right)$.

4. Design value of midspan absolute flow angle at each stage inlet and exit station $\left(\alpha_{1}\right.$ and $\left.\alpha_{2}\right)$.

Once this information is available, the stage stacking procedure is initiated by specifying the stage 1 inlet flow coefficient $\varphi_{l}$, inlet total pressure $P_{l}$, inlet total temperature $T_{l}$, and compressor corrected speed $N_{c}$. Selection of these parameters sets the values of $\psi, \eta, k_{b, 1}$, and $k_{b, 2}$ for stage 1 according to the stage 1 performance curves. With these parameters known, Eqs. (2-11) can be rearranged to solve for the stage 1 exit flow coefficient $\varphi_{2}$, as detailed in [11, 12].

For a stage without bleed, such as stage 1, the exit station and the inlet station of the downstream stage defined at an identical location (i.e. midgap), and thus $\varphi_{2}$ of this stage is equal to the inlet flow coefficient of the following stage. This value of stage 2 inlet flow coefficient, along with the specified $\mathrm{N}_{\mathrm{c}}$, sets the values of $\psi, \eta, \mathrm{k}_{\mathrm{b}, 1}$, and $\mathrm{k}_{\mathrm{b}, 2}$ for stage 2 according to its performance curves. This process is repeated for each subsequent stage through the entire machine, yielding an estimate of the overall performance of the compressor. Note that for stages neighboring interstage bleeds, the exit station of the upstream stage is at a different location than the inlet station of the downstream stage. Thus, the value of $\varphi_{2}$ is corrected to account for mass flow removal due to the bleed and for any change in annulus area between the exit and inlet stations.

\section{Constructing Stage Performance Curves}

The stage stacking procedure defined above requires, among other information, the fully defined performance curves of every stage in the compressor. During preliminary design, the performance curves are generally estimated from empirical

4

This material is declared a work of the U.S. Government and is not subject to copyright protection in the United States. Approved for public release; distribution is unlimited. 
correlations derived from test data. These correlations are well grounded near design point. However, at off-design conditions, their ability to estimate correct levels of performance is very often found to be lacking. In the current work, the stage performance curves were constructed using flow information extracted from APNASA CFD simulations. This database of CFD simulations had to be sufficiently extensive to allow a stage stacking method to be used to estimate compressor performance far from the design point. Simulations of the entire compressor will not, in general, yield the information required to fully populate the performance curves for all of its single stages. This is because certain stages will stall or choke, depending on operating conditions, well before others due to the way the stages are aerodynamically matched. In order to fully define the near-stall and near-choke portions of the characteristics for each stage, it is necessary to simulate small groups of stages in isolation.

Since stage characteristics were constructed from simulations of small groups of stages, it was necessary to show that stage characteristics constructed from these simulations matched those constructed from simulations of the entire compressor. To this end, a design speed simulation of stages 1 through 6 was generated and throttled along a speed line. Stage performance curves constructed from this simulation were compared to those constructed from a simulation of the entire compressor at design speed. The stage 1 performance curves of these two simulations are shown in Figure 2.
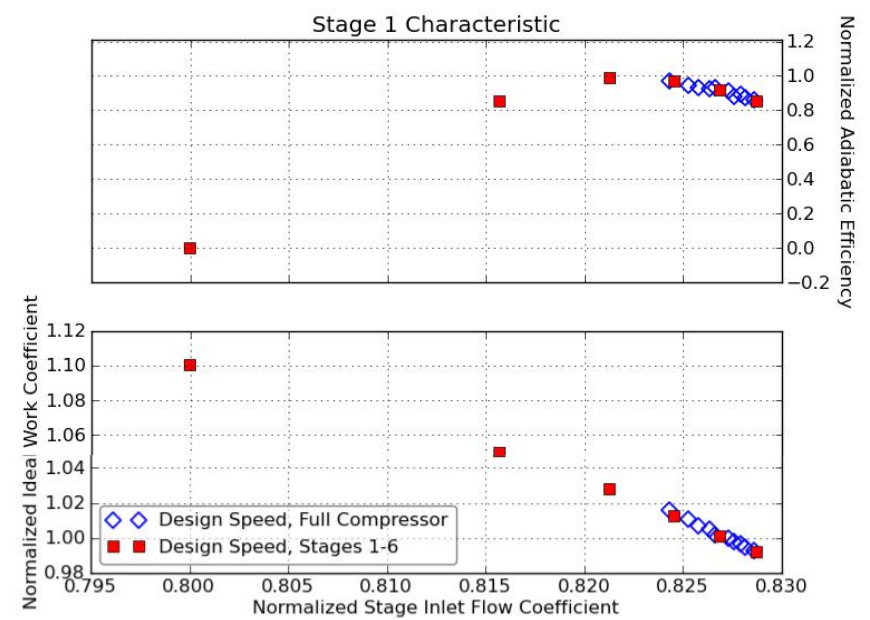

FIGURE 2: STAGE 1 PERFORMANCE CURVES AT DESIGN SPEED - FULL COMPRESSOR VERSUS STAGES 1-6

Points shown as open diamonds track the performance of stage 1 in the full compressor simulation and points shown as solid squares track the performance of stage 1 in a simulation of stages 1 through 6 only. The left-most points from both sets of data correspond to the near-stall points of the two simulations. Two major points are to be made from the results shown. Firstly, it is clear that the stage 1 characteristic constructed from the simulation of stages 1 through 6 lays on top of the stage 1 characteristic constructed from the full compressor simulation.
Therefore, it is valid to use results from simulations of groups of stages in place of full compressor simulations for the purposes of generating stage performance curves. Secondly, it is evident that simulations of the full compressor cannot adequately simulate the near-stall behavior of stage 1 . At design speed, the full compressor simulation goes into numerical stall at a much greater flow coefficient than the simulation of stages 1 through 6 . This is because a stage downstream of stage 6 is thought to be the stall limiting stage at design speed. Exclusion of this stage in the simulation of stages 1 through 6 allows stage 1 to be throttled to much lower flow coefficients as compared to the full compressor simulation. This demonstrates the need to simulate smaller groups of stages, rather than the full compressor, in order to fully populate the stage characteristics.

Another example showing the limited flow range for a stage simulated within the full compressor is seen in Figure 3. This figure plots the performance of stage 10. Open diamonds show the performance of stage 10 within full compressor simulations, which are unable to establish the choking flow coefficient of stage 10. The choking flow coefficient was instead determined from simulations of stages 8 through 10 in isolation, as shown by the solid squares in Figure 3. Choking of the stage is identified by the near-vertical slope of the efficiency curve at high flow coefficient.

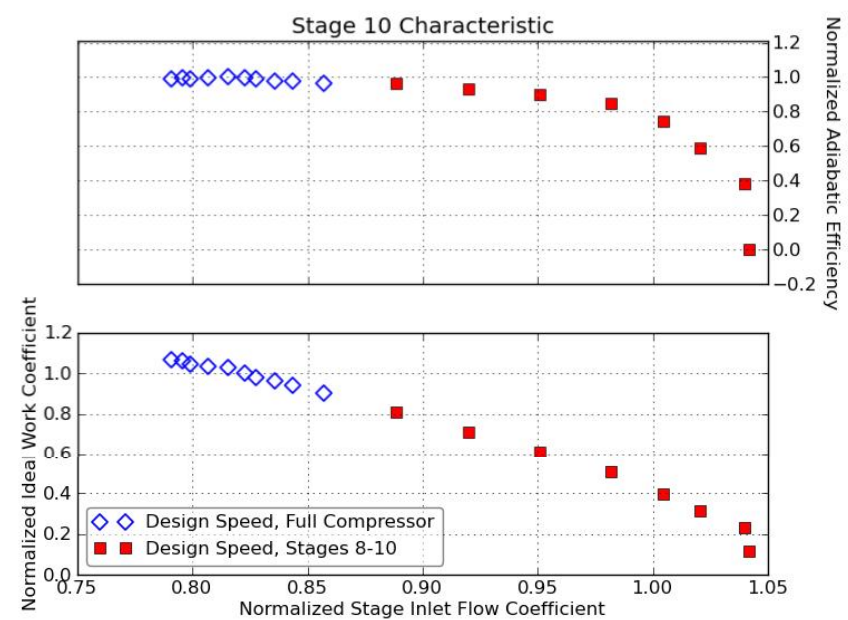

FIGURE 3: STAGE 10 PERFORMANCE CURVES AT DESIGN SPEED - FULL COMPRESSOR VERSUS STAGES 8-10

In simulating a group of embedded stages (e.g. stage 10) in this manner, it became necessary to include at least two stages upstream of the stage of interest. In [12], it was shown that the two upstream stages act as flow generators, which establish the multistage environment for the stage of interest. This is consistent with industry practices. Two stages upstream were adequate for generating the correct inlet conditions to the stage of interest as the simulation was throttled. Additionally, some simulations included at least one stage downstream, but it was found that in many cases, the downstream stage could be

This material is declared a work of the U.S. Government and is not subject to copyright protection in the United States. Approved for public release; distribution is unlimited. 
removed without impacting the characteristic curves, as shown in [12].

The stage performance curves generated in this manner displayed results consistent with [11]. As noted in [11], stages with inlet relative Mach number greater than about 0.75 have discrete curves, $\psi$ and $\eta$ as a function of $\varphi_{1}$, at different corrected speeds. In the current compressor, stages 1 through 4 have inlet Mach number greater than 0.75. Figure 4 shows the performance curves for stage 1 , which exhibit this dependence on corrected speed and on $\varphi_{1}$.
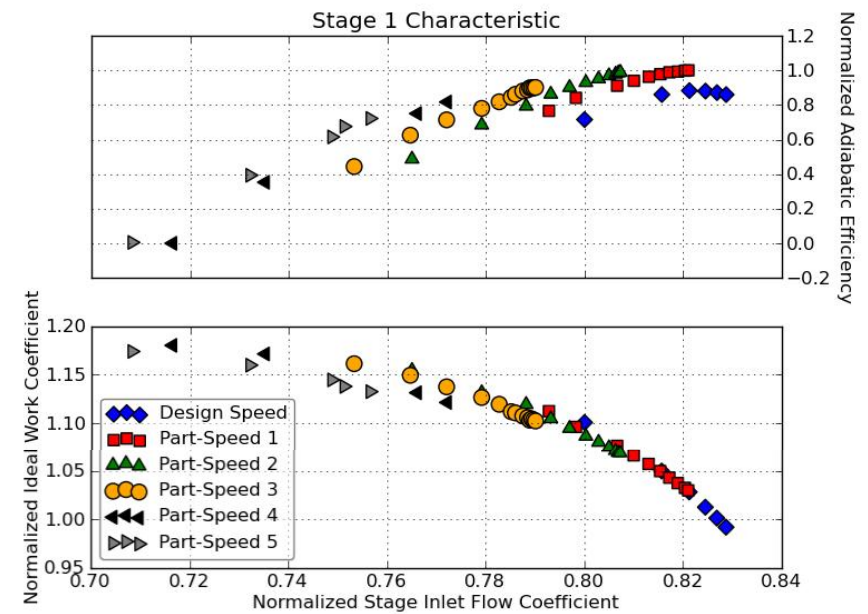

FIGURE 4: STAGE 1 PERFORMANCE CURVES AT VARIOUS CORRECTED SPEEDS

Furthermore, as noted in [11], stages with inlet relative Mach number less than 0.75 have performance curves which collapse to a single line irrespective of corrected speed. This was observed for stages 5 through 13, which have inlet relative Mach numbers less than 0.75 . The collapse of these curves is shown in Figure 5, which shows the performance curves of stage 5 .

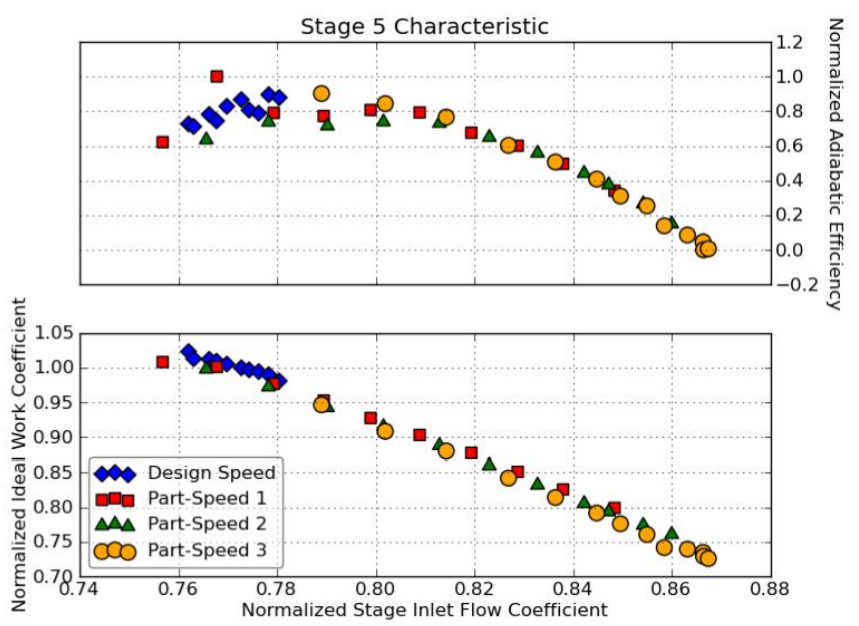

FIGURE 5: STAGE 5 PERFORMANCE CURVES AT VARIOUS CORRECTED SPEEDS
It is clear from Figure 5 that the performance curves for this stage have negligible dependence on corrected speed. Thus, performance curves of stages 1 through 4 were generated at various corrected speeds, whereas the performance curves of stages 5 through 13 could be fully populated with only design speed simulations.

\section{Collapse of Blockage Curves}

A noteworthy result observed when constructing the stage performance curves was the behavior of the blockage parameter $k_{b}$. When plotted as a function of $\varphi_{1}$ this parameter, like $\psi$ and $\eta$, showed a dependence on corrected speed for those stages with inlet relative Mach number greater than 0.75 (i.e. stages 1 through 4). This is shown in Figure 6, which plots the effective blockage at the exit of stage $1 k_{b, 2}$ as a function of stage 1 inlet flow coefficient $\varphi_{1}$ for various corrected speeds. It should be noted that the blockages shown here have been normalized by the value of stage 1 blockage near the design point.

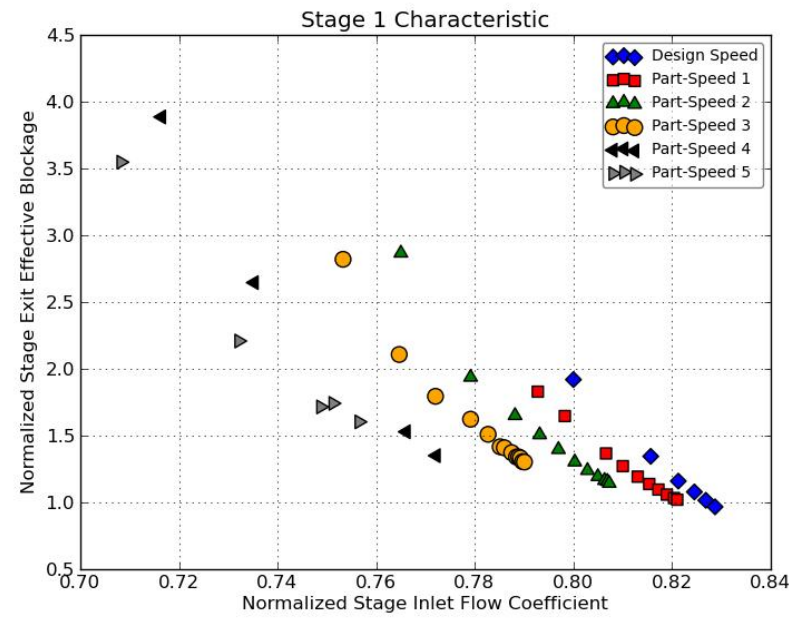

FIGURE 6: STAGE 1 BLOCKAGE CURVES AT VARIOUS CORRECTED SPEEDS

Furthermore, these blockage curves collapsed to a single line for those stages with inlet relative Mach number less than 0.75 (i.e. stages 5 through 13) irrespective of corrected speed. This is shown in Figure 7, which shows the stage 5 exit blockage curves at various corrected speeds.

This material is declared a work of the U.S. Government and is not subject to copyright protection in the United States. Approved for public release; distribution is unlimited. 


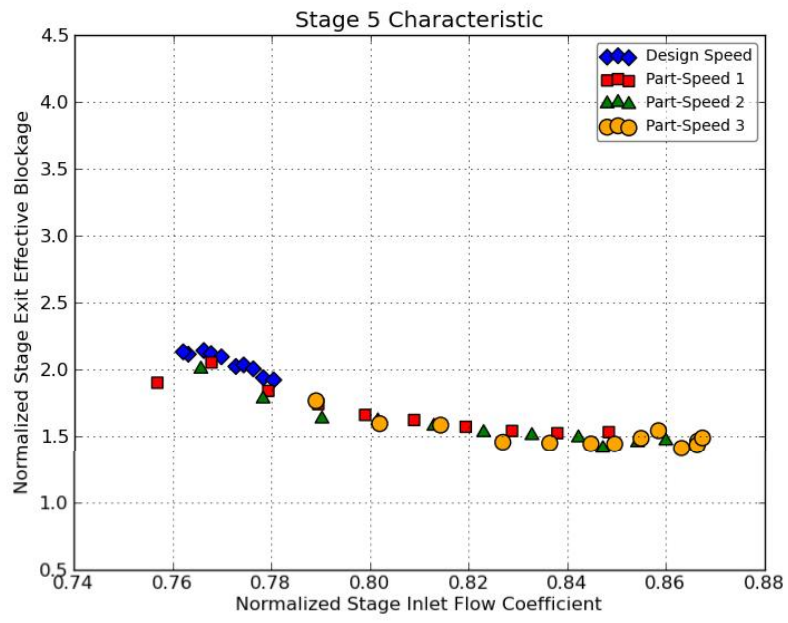

FIGURE 7: STAGE 5 BLOCKAGE CURVES AT VARIOUS CORRECTED SPEEDS

This correlation of blockage with flow coefficient and corrected speed is a result which, to the authors' knowledge, has not been published in the open literature. This correlation allowed for the implementation of the blockage curves into the stage stacking procedure described in [11], whereby the selection of inlet flow coefficient provided values of effective blockage in addition to values of the ideal work coefficient and adiabatic efficiency of a stage.

A heuristic argument is made here to understand the collapse of these blockage curves. As shown in [12], the blockage parameter was shown to be consistent with an existing blockage metric defined by Khalid, et al. [13] over a wide range of operating conditions. The blockage parameter defined in [13] is a measure of regions of flow where the gradient of a velocity component is large. This is the same region of flow where the dissipation function, and thus the entropy production, is large. It can be shown from the equation of efficiency, Eq. (5), that the entropy production for stages with Mach number less than 0.75 is only a function of flow coefficient. Thus, one may conclude that the collapse of the blockage curves as shown in the present work is a generic result.

\section{APPLICATIONS OF STAGE STACKING}

The typical use of stage stacking methods is to generate stage and overall performance estimates for a given compressor at specified operating conditions. In the current work, the stage stacking procedure was used to estimate off-design compressor performance at $97 \%$ speed, as detailed in [12]. These overall compressor performance estimates yielded total pressure ratio to within $1.6 \%$ and adiabatic efficiency within 0.3 points of CFD simulations of the entire compressor. The comparison of CFD and stage stacking results was done as a plausibility check on the stage stacking procedure.

In addition, the stage stacking procedure was used to estimate the minimum corrected speed for stable compressor operation with fixed IGV/VGV angles and fixed bleed rates. The details of this study follow.

\section{Estimation of Minimum Stable Corrected Speed}

The stage stacking procedure was used to estimate the minimum corrected speed which allowed stable compressor operation with geometry and bleed rates fixed. This was done by performing the stage-by-stage calculation, described previously, and picking near-stall values of stage 1 inlet flow coefficient along the various performance curves at different corrected speeds. The goal of the stage stacking calculations was to find the corrected speed which would result in a choking flow coefficient for the last stage in the compressor. Choked flow in the rear stage is typical of a compressor operating in isolation near minimum speed. After iteratively reducing corrected speed in the stage stacking calculations, it was found that operation at part-speed "N\%" was characterized by flow coefficients near stall at the compressor inlet, and near choke at the compressor exit. Part-speed N\% was found to be a corrected speed significantly less than design speed.

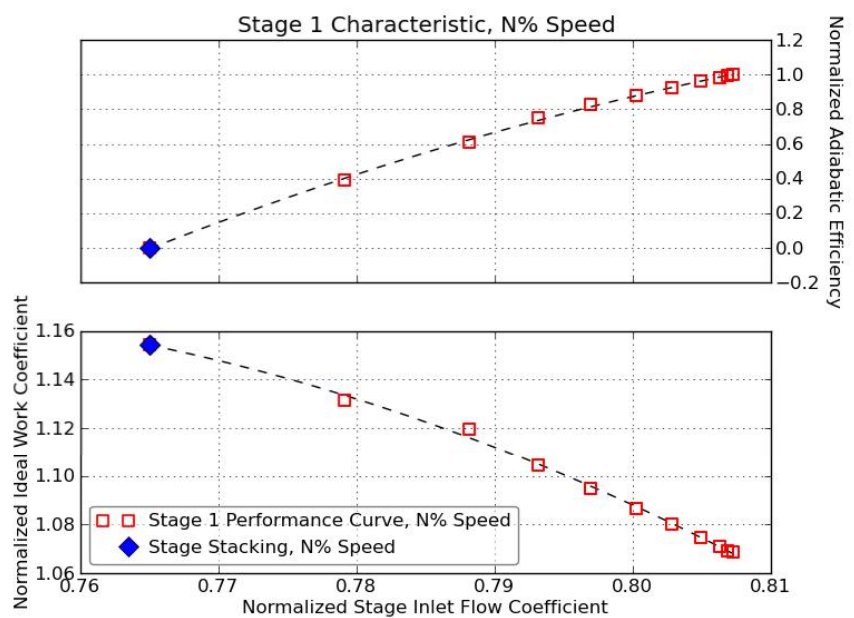

\section{FIGURE 8: STAGE 1 PERFORMANCE CURVES WITH STAGE STACKING ESTIMATE AT N\% SPEED}

Figure 8 shows the stage $1 \mathrm{~N} \%$ performance points as open squares. The solid diamond depicts the stage stacking estimated performance for the stage. As shown, the minimum (near-stall) value of flow coefficient was specified for the stage 1 inlet flow coefficient in this stage stacking calculation. Selection of this flow coefficient determined the ideal work coefficient, adiabatic efficiency, and effective blockage according to the stage performance 1 curves. The stage-bystage calculation was performed, resulting in a near-choke value of stage 13 inlet flow coefficient, as shown in Figure 9.

This material is declared a work of the U.S. Government and is not subject to copyright protection in the United States. Approved for public release; distribution is unlimited. 


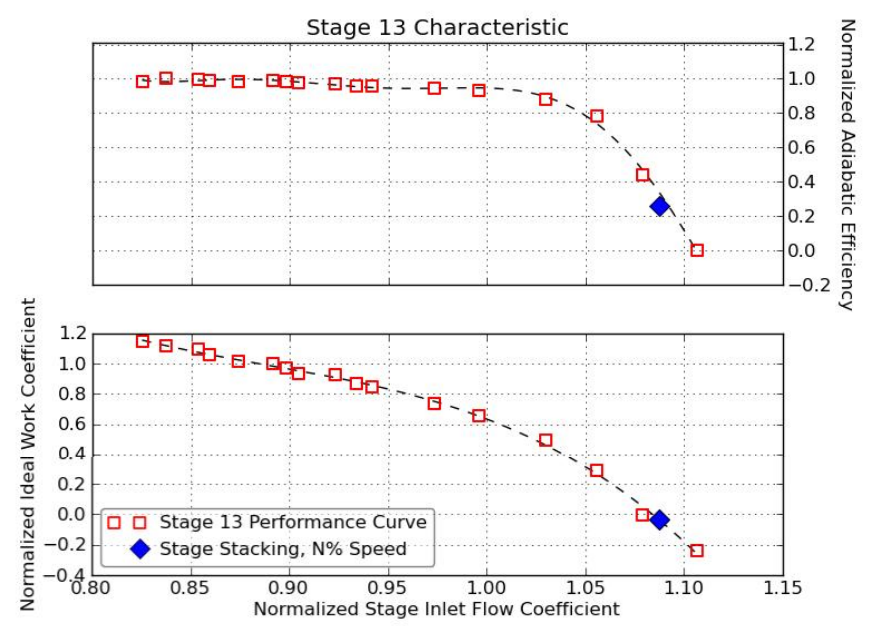

\section{FIGURE 9: STAGE 13 PERFORMANCE CURVES WITH STAGE STACKING ESTIMATE AT N\% SPEED}

A separate stage stacking calculation was performed for part-speed "N2\%", which is a corrected speed $2 \%$ less than part-speed N\%. A near-stall value of stage 1 inlet flow coefficient was picked along the $\mathrm{N} 2 \%$ speed stage 1 performance curves to initiate the stage stacking calculation, as shown in Figure 10.

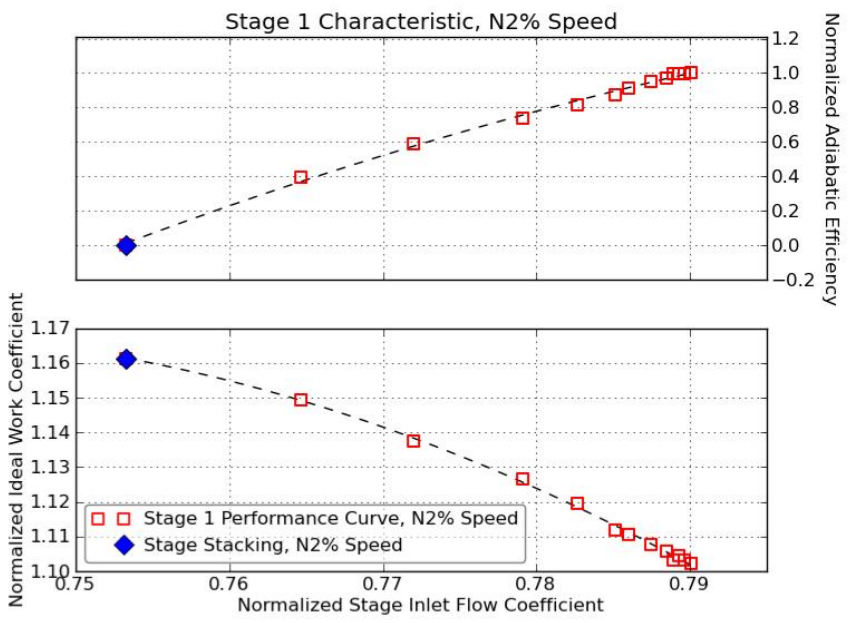

\section{FIGURE 10: STAGE 1 PERFORMANCE CURVES WITH STAGE STACKING ESTIMATE AT N2\% SPEED}

As the stage-by-stage calculation progressed through the machine, it was noted that the stage stacking estimate of stage 9 performance indicated that this stage was nearly choked, as shown in Figure 11. This is readily seen on the efficiency plot, which shows that the stage stacking point is on the near-vertical portion of the curve.

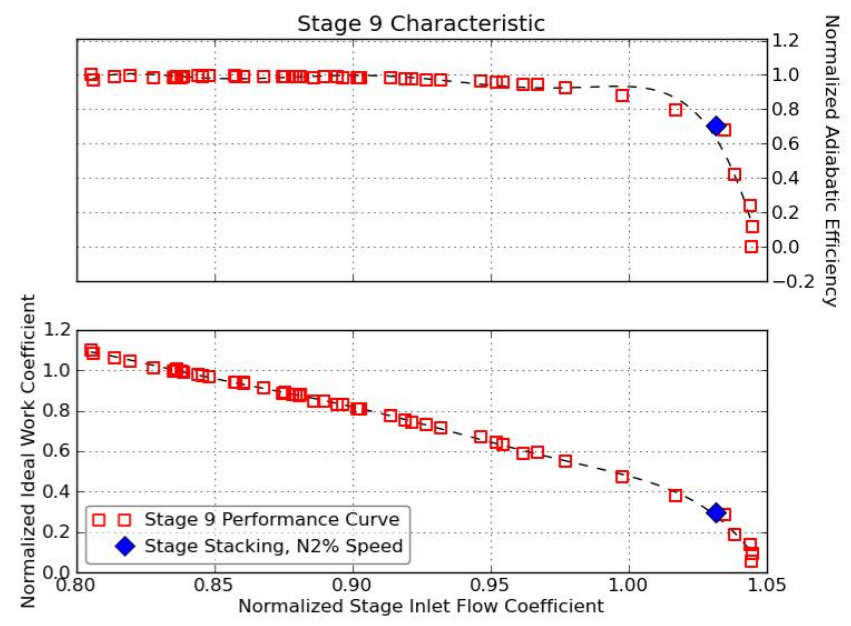

\section{FIGURE 11: STAGE 9 PERFORMANCE CURVES WITH STAGE STACKING ESTIMATE AT N2\% SPEED}

Attempting to continue the stage stacking procedure beyond stage 9 proved to be futile. This was because the stage 10 inlet flow coefficient estimated by the stage stacking procedure was greater than the maximum possible value attainable by the stage, as shown in Figure 12.

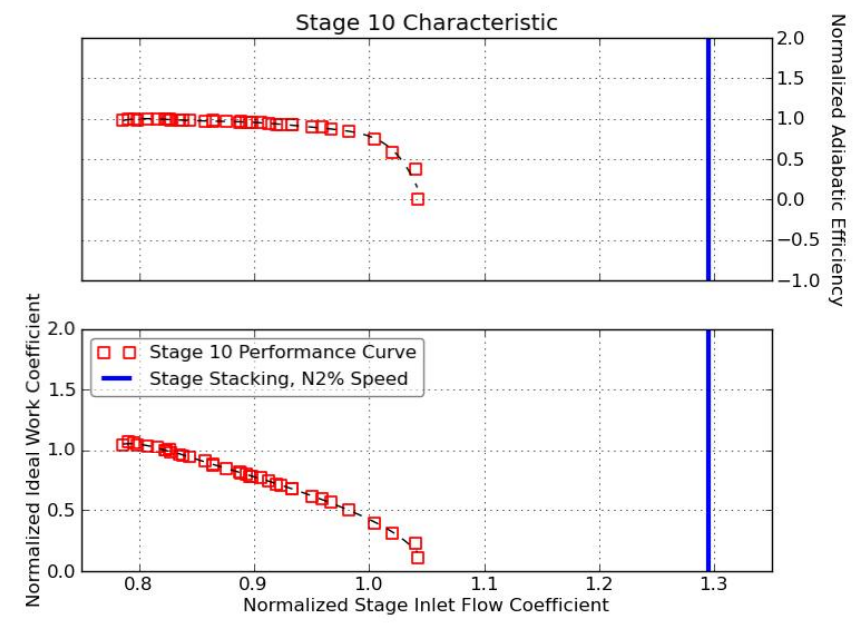

\section{FIGURE 12: STAGE 10 PERFORMANCE CURVES WITH STAGE STACKING ESTIMATE AT N2\% SPEED}

The stage 10 inlet flow coefficient estimated via stage stacking was far greater than the maximum, or choking, flow coefficient. Stage 10 cannot operate at such a high flow coefficient. In order to achieve operability at part-speed N2\%, the stage 10 inlet flow coefficient must decrease. For fixed geometry and bleed rates, this is only possible if the compressor inlet flow coefficient is reduced. However, a near-stall value of stage 1 inlet flow coefficient was specified in this case. The compressor would likely stall if it was to operate at a lower inlet flow coefficient. Hence at part-speed N2\%, there is no operating range for this compressor for bleed rates and IGV/VGV settings fixed to their nominal values. Thus, the

8

This material is declared a work of the U.S. Government and is not subject to copyright protection in the United States. Approved for public release; distribution is unlimited. 
minimum stable speed of the compressor is estimated to be greater than $\mathrm{N} 2 \%$, and equal to or less than N\% speed. The final example, which follows, details a bleed sensitivity study.

\section{Operability and Bleed Sensitivity Study}

A bleed sensitivity study was conducted to estimate the stage 5 bleed rate required such that stage 13 operated nearchoke, and all other stages operated within their stable operating ranges at part-speed N2\%. This was accomplished by iteratively varying stage 5 bleed rate within the stage stacking procedure and performing the stage-by-stage calculation with the stage 1 inlet flow coefficient fixed to its near-stall value for N2\% speed. These iterations were performed until the stage stacking procedure estimated a near-choke flow coefficient for stage 13, with all upstream stages operating in their stable operating ranges. The resulting stage 5 bleed rate was approximately 7 times greater than the nominal rate.

The value of exit static pressure resulting from the stage stacking estimate was specified as the exit static pressure for a CFD simulation at N2\% speed using the new value of stage 5 bleed rate, as detailed in [12]. Stage performance parameters calculated from this CFD simulation were then compared to the stage stacking estimate. Figure 13 shows the stage 1 performance curves.

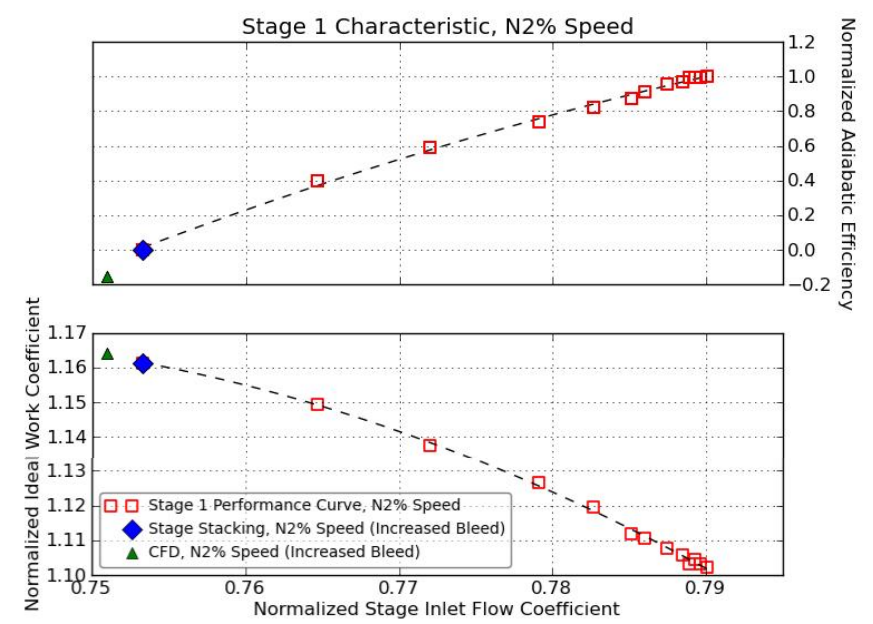

FIGURE 13: STAGE 1 PERFORMANCE CURVES WITH STAGE STACKING AND CFD RESULTS AT N2\% SPEED

The near-stall value of flow coefficient is denoted by the solid diamond. The CFD simulation is shown as a solid triangle in Figure 13, and it indicates that the CFD simulation converged to a slightly lower stage 1 inlet flow coefficient at N2\% speed as compared to the stage stacking estimate. The result of increasing the stage 5 bleed rate for stage 9 is shown in Figure 14.

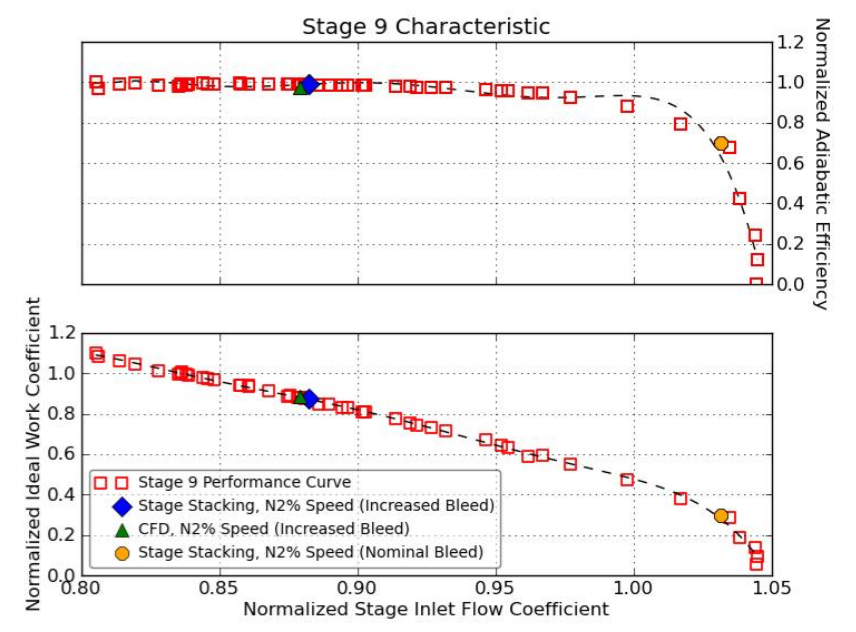

FIGURE 14: STAGE 9 PERFORMANCE CURVES WITH STAGE STACKING AND CFD RESULTS AT N2\% SPEED

The stage stacking estimate of stage 9 inlet flow coefficient with nominal stage 5 bleed rate (shown as a solid circle) is compared to that with increased stage 5 bleed rate (shown as a solid diamond). The increase in stage 5 bleed rate resulted in a significantly reduced estimate of stage 9 inlet flow coefficient. Examining the stage 13 performance curves in Figure 15 shows that the CFD simulation resulted in stage 13 being choked, which was the goal of the bleed sensitivity study.

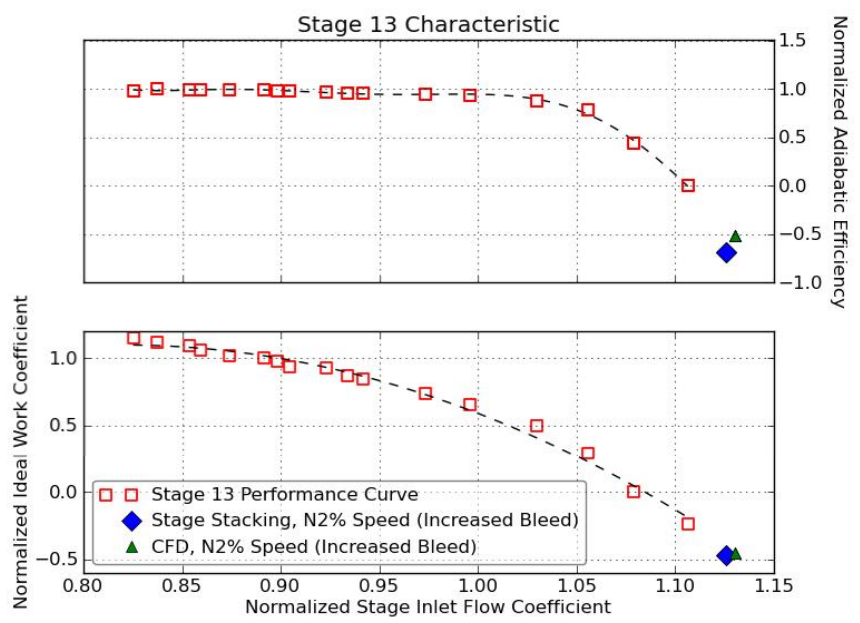

FIGURE 15: STAGE 13 PERFORMANCE CURVES WITH STAGE STACKING AND CFD RESULTS AT N2\% SPEED

Thus, the stage stacking procedure is able to describe a bleed schedule which could extend the operating range of this compressor down to part-speed N2\%, which is a corrected speed $2 \%$ lower than N\% speed. Increasing the stage 5 bleed rate in the compressor acted to reduce the flow coefficient for choked stages within the middle of the machine. The stage stacking calculation estimated the compressor inlet corrected flow to within $0.3 \%$ of the resulting CFD simulation. Overall total pressure ratio was estimated to within $1.3 \%$ of the CFD

This material is declared a work of the U.S. Government and is not subject to copyright protection in the United States. Approved for public release; distribution is unlimited. 
result, and overall adiabatic efficiency was estimated to within 0.42 points of the CFD result.

As a final step in this operability study, the stage 5 bleed rate in the CFD simulation was reduced from 7 times the nominal value back down to the nominal value. This resulted in numerical stall in the front stages of the compressor. This result corroborated the estimated minimum stable corrected speed from the stage stacking calculations, as there was no operating range at part-speed $\mathrm{N} 2 \%$ with nominal IGV/VGV and bleed settings.

\section{CONCLUSIONS}

The major conclusions of this research effort can be summarized as follows:

- A modified stage stacking procedure was developed which attempted to account for flow blockage through a novel mathematical closure on static pressure to calculate effective flow area.

- The blockage term, like the ideal work coefficient and adiabatic efficiency, was found to be a function of:

$\circ$ inlet flow coefficient and corrected speed, for those stages with inlet relative Mach number greater than 0.75 (stages 1-4).

$\circ$ inlet flow coefficient only, for those stages with inlet relative Mach number less than 0.75 (stages 5-13).

- To fully define the stage performance curves at the nearstall and near-choke sides using CFD results, groups of stages must be simulated in isolation. The complete flow range of a given stage cannot, in general, be modeled in simulations of the full compressor.

- The stage stacking procedure yielded:

o good off-design estimates of overall compressor performance as compared to CFD simulations.

o a good estimate for the minimum corrected speed for stable operation as compared to experiment.

o a good estimate of a bleed schedule to expand the partspeed operability of the compressor as compared to CFD simulations.

The primary use of the proposed stage stacking procedure would be during the design of a compressor which implements incremental changes on an existing design; for example, when the designer is interested in the effect on overall compressor performance after changing bleed flow rates, IGV setting angles, or blade shape of a single stage.

\section{ACKNOWLEDGMENTS}

The authors would like to thank Dr. Matthew D. Montgomery, Dr. Eric E. Donahoo, Mr. Paul S. Spedaler, Mr. David Wasdell, and Dr. Christian Cornelius of Siemens Energy, Inc. and Dr. Choon S. Tan of the Massachusetts Institute of Technology for useful suggestions during many technical discussions throughout the course of this work. The authors would like to acknowledge Mr. Richard A. Mulac of NASA Glenn Research Center for his technical assistance in running the CFD and meshing software. The first author would like to acknowledge funding of this work by the NASA Graduate
Student Researchers Program (GSRP) through a Reimbursable Space Act Agreement between NASA Glenn Research Center and Siemens Energy, Inc.

\section{REFERENCES}

[1] Kiesow, H. J. and McQuiggan, G., 2007, "The Challenges Facing the Utility Gas Turbine," ASME Turbo Expo, Paper No. GT2007-27180.

[2] Chaker, M. and Meher-Homji, C. B., 2007, "Evaporative Cooling of Gas Turbine Engines - Climatic Analysis and Application in High Humidity Regions," ASME Turbo Expo, Paper No. GT2007-27866.

[3] Wright, P. I. and Miller, D. C., 1991, "An Improved Compressor Performance Prediction Model," IMechE Paper No. C423/028, pp. 69-81.

[4] Hearsey, R. M., 2007, "Program HT0300 Vol. II: Theory \& Methods," Program HT0300 User Guide.

[5] Casey, M. and Robinson, C., 2010, "A New Streamline Curvature Throughflow Method for Radial Turbomachinery," ASME J. of Turbomachinery, Vol. 132.

[6] Mulac, R. A., 1986, "A Multistage Mesh Generator for Solving the Average-Passage Equation System," NASA CR-179539.

[7] Adamczyk, J. J., Celestina, M. L., Beach, T. A., and Barnett, M., 1990, "Simulation of Three-Dimensional Viscous Flows Within a Multistage Turbine," ASME J. of Turbomachinery, Vol. 112, pp. 370-376.

[8] Adamczyk, J. J., 1985, "Model Equation for Simulating Flows in Multistage Turbomachinery," ASME 85-GT-226.

[9] Adamczyk, J. J., Mulac, R. A., Celestina, M. L., 1986, “A Model for Closing the Inviscid Form of the AveragePassage Equation System," ASME 86-GT-227.

[10]Celestina, M. L., 1999, "Segmented Domain Decomposition Multigrid for 3-D Turbomachinery Flows," $\mathrm{Ph} . \mathrm{D}$. Thesis, Department of Aerospace Engineering and Engineering Mechanics, University of Cincinnati.

[11]Robbins, W. H. and Dugan Jr., J. F., 1965, "Prediction of Off-Design Performance of Multistage Compressors," Aerodynamic Design of Axial-Flow Compressors, NASA SP-36.

[12] Kulkarni, S., 2012, "Development of a Methodology to Estimate Aero-Performance and Aero-Operability Limits of a Multistage Axial Flow Compressor for Use in Preliminary Design," M.S. Thesis, Department of Mechanical and Aerospace Engineering, Case Western Reserve University.

[13] Khalid, S. A., Khalsa, A. S., Waitz, I. A., Tan, C. S., Greitzer, E. M., Cumpsty, N. A., Adamczyk, J. J., and Marble, F. E., 1999, "Endwall Blockage in Axial Compressor," ASME J. of Turbomachinery, Vol. 121, pp 449-509.

10

This material is declared a work of the U.S. Government and is not subject to copyright protection in the United States. Approved for public release; distribution is unlimited. 